\title{
Delayed Recurrent Laryngeal Nerve palsy following total thyroidectomy: A case report
}

\author{
D.N. Goyal, Venkateswara Rao Katta, Vijay Kumar V.R.N, Sumeera Farhath, \\ Aravinda Sagar K, Avinash G . \\ Department of General Surgery, MNR Medical College and Hospital, Sangareddy, Andhra Pradesh, India.
}

\begin{abstract}
We report a case of delayed recurrent laryngeal nerve palsy following total thyroidectomy. A 25 year old male patient presented to us with a left thyroid swelling. Investigations have revealed the presence of follicular neoplasm in the left thyroid nodule, which was measuring $6 \mathrm{cms}$ in diameter. An Indirect Laryngoscopy pre operatively has not revealed any abnormality. A total thyroidectomy was performed. Intra operatively, the left recurrent laryngeal nerve could not be identified, however at the end of surgery, visual confirmation of bilateral normal vocal cord movements was carried out. On fourth post-operative day, patient developed hoarse voice. An Indirect laryngoscopy has confirmed loss of movements in the left vocal cord and the patient was diagnosed to have delayed left recurrent laryngeal nerve palsy. The patient did not have any respiratory symptoms and received voice therapy. His voice returned to normal by three months.
\end{abstract}

Key Words:Recurrent Laryngeal Nerve Injury (RLNI), Total thyroidectomy, Nerve at Risk (NAR), Indirect Laryngoscopy (IDL), Unilateral Vocal Fold paralysis (UVFP), Indirect Laryngoscopy (IDL).

\section{Introduction:}

Routine visual identification of the RLN has been shown to result in a lower incidence of RLN injury in multiple studies[1-3]. But this operative standard still results in a $4 \%$ to $8 \%$ incidence of initial postoperative RLN paralysis; with continued follow up, the RLN frequently recovers function, resulting in a permanent paralysis rate of $1 \%$ to $2 \%[4-7]$. The most common laryngoscopic findings in unilateral vocal fold paralysis beyond vocal fold motion impairment include bowing, incomplete glottal closure, and phase asymmetry on videostroboscopy[14].The position of the vocal fold (eg, paramedian vs lateral) does not necessarily clarify the location of the lesion along the neurologic pathway from brain to motion of vocal fold[15]

\section{Case Report:}

We report a 25 year old male patient who presented to us with a gradually increasing swelling over the left thyroid region for one year. The swelling was small to start and gradually increased to its current size. There were no clinical or biochemical signs of hypo or hyper thyroidism. On examination, there was a left thyroid nodule measuring $6 \mathrm{cms}$ in diameter, not moving with tongue protrusion. There were no palpable lymph nodes in the neck.

An ultrasound examination was carried out, which revealed 58 X 47 X $35 \mathrm{~mm}$ hyperechoic heterogeneous space occupying lesion with multiple cystic spaces and mild increased vascularity noted with in the left lobe of thyroid.The patient then underwent a fine needle aspiration cytology which was reported as features suggestive of follicular neoplasm possibly adenoma.

An indirect laryngoscopy was performed and there was no abnormality reported. After counselling the patient about the significant finding of follicular neoplasm, we planned for a total thyroidectomy. Informed consent was obtained from the patient after explaining the risk of recurrent laryngeal nerve injury.

Intra operatively, the left lobe was meticulously dissected with release of adhesions posteriorly. During surgery, the left recurrent laryngeal nerve could not be identified. We then proceeded to a completion total thyroidectomy by dissecting the remaining thyroid gland. The right recurrent laryngeal nerve was identified during the procedure. During extubation, normal position of both vocal cords was confirmed with laryngoscopy. Post operatively the patient had normal voice for the first three days. On fourth post-operative day, patient complained of hoarse voice. There were no significant respiratory complaints. An indirect laryngoscopy confirmed left vocal cord paralysis with loss of movements with in the left vocal cord. The right vocal cord was showing normal movements. Patient was referred for voice therapy. This included abdominal breathing and humming/resonant voice to encourage closure of glottis. After three months of voice therapy patient reported improvement in voice quality and an indirect laryngoscopy has confirmed normal movements in both vocal cords. The final histopathology of the specimen was that of a colloid goiter with features suggestive of follicular adenoma. 


\section{Discussion:}

The risk of recurrent laryngeal nerve injury is increased with thyroidectomy for cancer, sub sternal goiter, chronic thyroiditis, Graves' disease, and re-operative neck surgery[4].These disease processes make RLN identification more difficult. Injury to the RLN can result from sharp trauma (transection), clamping, ligation, compression, traction, thermal injury, or ischemia[8]. The anterior motor branch of a recurrent laryngeal nerve bifurcating near the ligament of Berry is particularly at risk of traction injury[9].Laryngoscopy is an essential part of the evaluation of UVFP. Our case is unique in that there was delayed presentation of recurrent laryngeal nerve palsy on the fourth post-operative day, which has recovered completely in three months' time.We believe vascular spasm as a possible cause for this delayed presentation of vocal cord palsy. Voice therapy in UVFP is typically directed at abdominal breathing and humming/ resonant voice to improve closure of the glottis, encourage abdominal breath support, and improve vocal fold function while avoiding supraglottic hyper function[10]. Significant numbers of patients with UVFP who opted for voice therapy reported vocal improvement subjectively or as measured by glottal closure, acoustic measurements, pitch range, and/or patientreported voice handicap[11, 12].Interpretation of the impact of voice therapy in UVFP may be obscured by returning neurologic function [13]and it is unknown whether there is a relationship between voice therapy and neurologic recovery[10].

\section{References:}

[1] Jatzko GR, Lisborg PH, Muller MG, et al. Recurrent nerve palsy after thyroid operations - principal nerve identification and a literature review. Surgery 1994;115:139-144.

[2] Wagner HE, Seiler C. Recurrent laryngeal nerve palsy after thyroid gland surgery. Br J Surg 1994;81:226-228

[3] Hermann M, Alk G, Roka R, et al. Laryngeal recurrent nerve injury in surgery for benign thyroid disease: effect of the nerve dissection and impact of individual surgeon in more than 27,000 nerves at risk. Ann Surg 2002;235:261-268

[4] Randolph GW. Surgical anatomy of the recurrent laryngeal nerve. In: Randolph GW, ed. Surgery of the thyroid and parathyroid glands. Philadelphia, PA: Saunders; 2003:300-342.

[5] Chiang FY,Wang LF,Huang YF, et al. Recurrent laryngeal nerve palsy after thyroidectomy with routine identification of the recurrent laryngeal nerve. Surgery 2005; 137:342-347.

[6] ChanW, Lang BH, Lo C.The role of intraoperative neuromonitoring of the recurrent laryngeal nerve during thyroidectomy: a comparative study on 1000 nerves at risk. Surgery 2006;140: 866-873.

[7] Aytac B, Karamercan A. Recurrent laryngeal nerve injury and preservation in thyroidectomy. Saudi Med J 2005;26:1746- 1749.

[8] Myssiorek D. Recurrent laryngeal nerve paralysis: anatomy and etiology. Otolaryngol Clin North Am 2004;37:25-44.

[9] K Snyder, T C Lairmore, J C Hendricks, J W Roberts. Elucidating Mechanisms of Recurrent Laryngeal Nerve Injury during Thyroidectomy and parathyroidectomy. J Am Coll Surg 2008; 206:123-130.

[10] S Misono, A L Merati. Evidence-Based Practice. Evaluation and Management of unilateral vocal fold paralysis. Otolaryngol Clin N Am 45 (2012) 1083-1108.

[11] D'Alatri L, Galla S, Rigante M, et al. Role of early voice therapy in patients affected by unilateral vocal fold paralysis. J Laryngol Otol 2008; 122(9):936-41.

[12] Heuer RJ, Thayer Sataloff R, Emerich K, et al. Unilateral recurrent laryngeal nerve paralysis: the importance of "preoperative" voice therapy. $J$ Voice 1997; 11(1):88-94.

[13] Mattioli F, Bergamini G, Alicandri-Ciufelli M, et al. The role of early voice therapy in the incidence of motility recovery in unilateral vocal fold paralysis. Logoped Phoniatr Vocol 2011; 36(1):40-7

[14] Wang W, Chen D, Chen S, et al. Laryngeal reinnervation using ansa cervicalis for thyroid surgery-related unilateral vocal fold paralysis: a long-term outcome analysis of 237 cases. PLoS One 2011; 6(4):e19128.

[15] Woodson GE. Configuration of the glottis in laryngeal paralysis. I: Clinical study. Laryngoscope 1993; 103(11 Pt 1):1227-34. 